\title{
Social influences on the identification of toxic foods by Norway rats
}

\author{
BENNETT G. GALEF, JR. \\ McMaster University, Hamilton, Ontario, Canada
}

\begin{abstract}
In the present experiments, a naive "observer" rat first interacted with a "demonstrator" rat previously fed a diet unfamiliar to the observer. The observer then sampled two unfamiliar diets, one of which was the diet its demonstrator had eaten. The observer was then injected with $\mathrm{LiCl}$ and, following recovery from toxicosis, was offered a choice between the two diets it sampled prior to toxicosis induction. It was found that: (1) each observer rat formed an aversion to whichever diet its demonstrator had not eaten, (2) effects of demonstrators on aversion learning by observers were present even if there was a 7- or 8-day delay between interaction of a demonstrator and observer and diet sampling by the observer, and (3) observers interacting with 3 demonstrators, each fed a different diet, subsequently exhibited a reduced tendency to form an aversion to each of the diets eaten by their demonstrators. Taken together, the results indicate that information acquired from conspecifics as to the diets they have eaten can play an important role in determining the foods to which otherwise naive rats will learn aversions.
\end{abstract}

In a recently reported study of the effects of social interaction on the identification of toxic foods by Norway rats (Galef, 1986a), a naive "observer" rat interacted with 2 conspecific "demonstrators" in succession; just before interacting with the observer, each demonstrator ate a food (Diet N1) that was unfamiliar to the observer. The observer then ate, one after the other, two unfamiliar foods (Diets N1 and N2). One of the two foods fed to the observer (Diet N1) was the same food both of its demonstrators had eaten. Following its meals of Diets $\mathrm{N} 1$ and $\mathrm{N} 2$, the observer was injected with $\mathrm{LiCl}$. Finally, after recovery from toxicosis, the observer was offered a choice between Diets N1 and N2 for $22 \mathrm{~h}$. During the 22-h choice test, observer rats ate much more of the food that their respective demonstrators had eaten (Diet N1) than of the alternative food available (Diet N2). Observers formed a stronger aversion to the food their demonstrators had not eaten (Diet N2) than to the food their demonstrators had eaten (Diet N1).

A plausible account of how such social influence on aversion learning might proceed is easily constructed. First, it has long been known that a naive rat that eats two diets before becoming ill will form a stronger aversion to the less familiar of the two diets it ate (Kalat \& Rozin, 1973; Revusky \& Bedarf, 1967). Second, results of recent studies suggest that an observer rat in contact with a recently fed conspecific demonstrator can use both olfactory and gustatory cues to identify the diet fed to its

Funding for this research was provided by grants from the Natural Sciences and Engineering Research Council of Canada and the McMaster University Research Board. I thank Christine Hughes, Cheryl Spencer, and Susan Madronich for their technical assistance and Mertice Clark, Harvey Weingarten, and Matthew Beck for critical reviews of previous drafts of the manuscript. Requests for reprints should be sent to Bennett G. Galef, Jr., Department of Psychology, McMaster University, Hamilton, Ontario L8S 4K1, Canada. demonstrator: Demonstrators emit diet-related odors from their digestive tracts that allow observers to identify the diet a demonstrator recently ate (Galef \& Stein, 1985). Particles of food cling to the pelage of a recently fed rat and provide both olfactory and gustatory cues that permit observer identification of the food a demonstrator has eaten (Galef \& Stein, 1985; Galef \& Wigmore, 1983; Posadas-Andrews \& Roper, 1983). Thus, exposure of a naive observer to demonstrators could make observers familiar with the food that its demonstrators ate. In consequence, interaction with a demonstrator that had eaten a food could act, as does actual ingestion of a food, to reduce the food's subsequent associability with toxicosis.

This tendency to attribute toxicosis more readily to a food that is completely unfamiliar than to a food experienced indirectly, through exposure to conspecifics that have eaten the food, may have important functional consequences. It is probably usually true that an unfamiliar food that others of one's social group are eating is less likely to be toxic than unfamiliar foods that others of one's social group are not eating (Galef, 1986a). Hence, a rat that ate more than one novel food before becoming ill could benefit by attributing its illness to that novel food it had eaten that others of its group had not eaten.

In the present series of experiments, I explored the range of circumstances in which exposure of observer rats to conspecific demonstrators can influence learning of aversions by the observers. The results are discussed in terms of possible contributions of social interaction to toxic food identification and avoidance by rats living, as rats do in natural circumstances, in social groups.

\section{EXPERIMENT 1}

In our first study of the effects of exposure to demonstrators fed a certain diet on subsequent formation of an 
aversion to that diet by observers (Galef, 1986a), observers interacted with demonstrators, ate novel foods, and suffered toxicosis in rapid succession. It seems likely that, in natural circumstances, some hours or days would pass between a naive individual's encounter with conspecifics that had eaten an unfamiliar food (Diet N1) and that naive individual's initiation of a foraging expedition during which it ate Diet N1, as well as other novel foods, one of which was toxic. If interaction with conspecifics plays a significant role in toxic diet identification by rats in a natural habitat, then interaction with a demonstrator fed a diet (N1) should influence aversion learning by an observer even if the observer does not eat unfamiliar diets (N1 and N2) and become ill for many hours after interacting with a demonstrator fed Diet N1.

Method
Subjects
Fifty-four experimentally naive 42-day-old female Long-Evans
rats obtained from Charles River, Canada (St. Constant, Quebec)
served as observers. An additional 54 56- to 63-day-old females
of the same strain, born and reared in the McMaster colony, served
as demonstrators. Observers and their respective demonstrators were
randomly assigned to the 0-, 1-, and 8-day delay conditions described
below.

\section{Apparatus}

Observers were housed individually throughout the experiment in $22 \times 24 \times 27.5 \mathrm{~cm}$ wire-mesh hanging cages. Demonstrators were housed individually in plastic shoe-box cages in a room separate from observers.

\section{Procedure}

Treatment of observers and demonstrators during the experiment was as follows (see Figure 1):

Step 1. Observers and demonstrators were introduced into their respective cages and placed on a $23-\mathrm{h}$ food-deprivation schedule, receiving powdered Purina Laboratory Rodent Chow (Diet Pur) for $1 \mathrm{~h} /$ day for 2 days.

Step 2. Following a third 23-h period of food deprivation, each demonstrator was offered, for $1 \mathrm{~h}$, a food cup containing either cocoa-flavored diet (Diet Coc; 27 demonstators) or cinnamonflavored diet (Diet Cin; 27 demonstrators). (Diet $\mathrm{Coc}=$ powdered Purina Laboratory Rodent Chow adulterated $2 \%$ by weight with Hershey's cocoa; Diet Cin = powdered Purina Laboratory Rodent Chow adulterated $1 \%$ by weight with McCormick's pure ground cinnamon.) Because the experiment required that each demonstrator eat the diet presented to it, demonstrators failing to eat $2.0 \mathrm{~g}$ of the diet offered them during Step $2(n=2)$ and their respective observers were discarded.

Step 3. Immediately following feeding of each demonstrator, it was introduced into the cage of an observer, and each demonstratorobserver pair was left undisturbed to interact freely for $30 \mathrm{~min}$.

Steps 4, 5, and 6. At the end of the 30-min period of interaction between demonstrator and observer, each demonstrator was removed from its observer's cage and, in the case of observers in the 0-delay condition $(n=9)$, was immediately replaced with a weighed food cup containing Diet Coc, which was left in each observer's cage for $15 \mathrm{~min}$. At the end of this first 15-min observer feeding period, the food cup containing Diet Coc was removed and immediately replaced with a second food cup containing a weighed sample of Diet Cin, which was also left in each observer's cage for $15 \mathrm{~min}$.

Observers in both 1-day-delay $(n=9)$ and 8-day-delay $(n=9)$ conditions were offered, immediately following removal of their respective demonstrators, a food cup containing powdered Purina Laboratory Rodent Chow for $1 \mathrm{~h}$. Observers in the 1-day-delay condition were fed Diets Coc and Cin (i.e., Steps 5 and 6 in Figure 1) 24 h after interacting with their respective demonstrators. Observers in the 8-day-delay condition were offered powdered Purina Laboratory Rodent Chow for $1 \mathrm{~h}$ /day for 7 days before being offered Diets Coc and Cin (Steps 5 and 6 in Figure 1).

Step 7. Immediately following termination of the $15 \mathrm{~min}$ of feeding on Diet Cin, each observer was injected intraperitoneally with $1 \%$ of body weight $1 \% \mathrm{w} / \mathrm{v} \mathrm{LiCl}$ solution.

Step 8. One hour following injection, pellets of Purina Laboratory Rodent Chow were placed in each observer's cage, and each was given $24 \mathrm{~h}$ to recover from the effects of toxicosis.
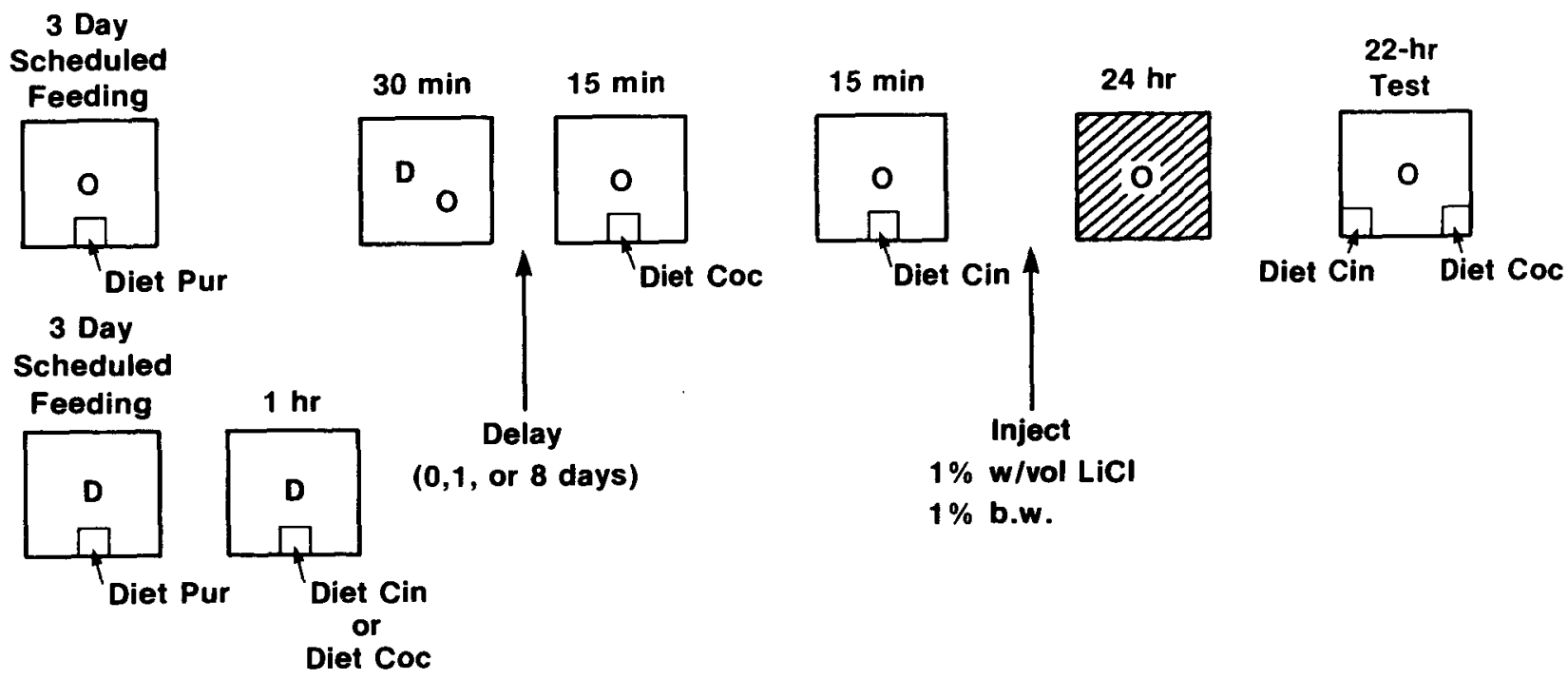
Step: 1
2
$3 \quad 4 \quad 5$
6
7
8
9
Figure 1. Schematic of the procedure of Experiments 1 and 2. Cross-hatching = ad-lib access to Purina Laboratory Rodent Chow; $\mathbf{D}=$ demonstrator; $\mathbf{O}=$ observer. 


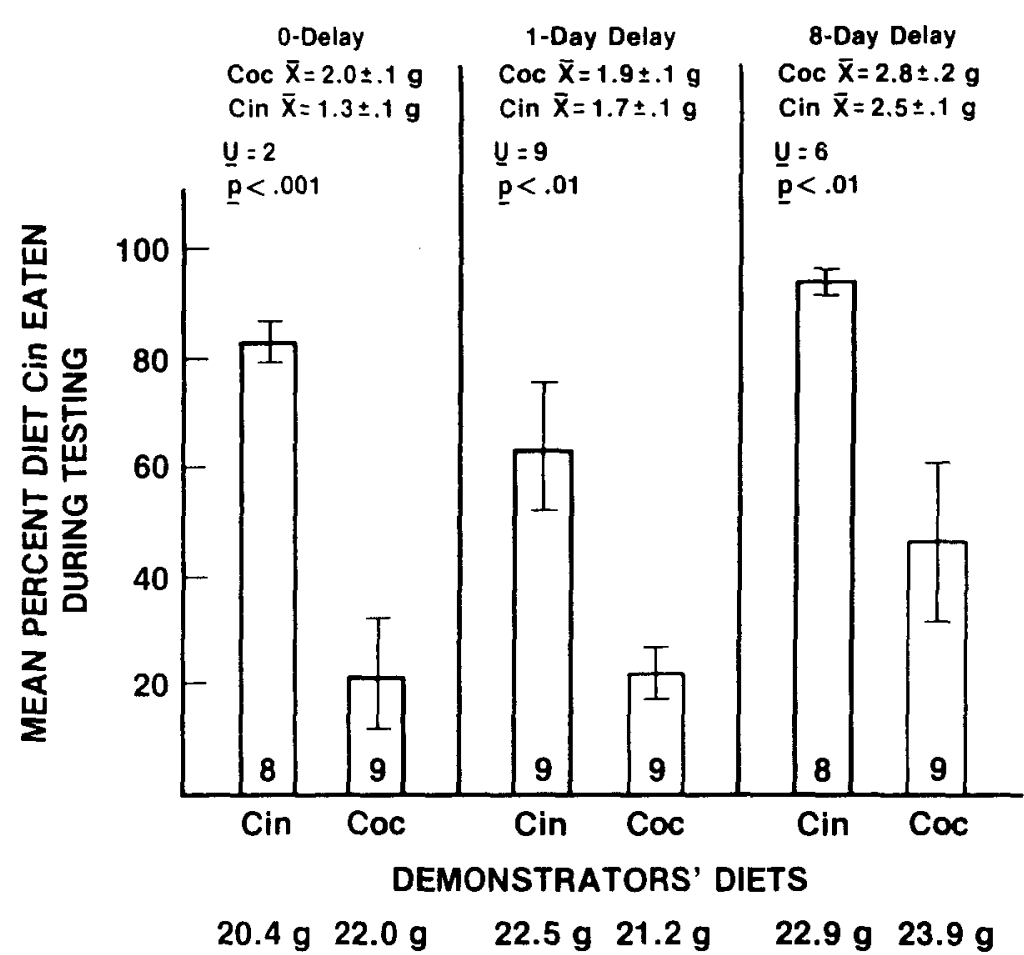

Figure 2. Mean amount of Diet Cin eaten by observers, as a percentage of total amount consumed during testing (Step 9 of Procedure). Numerals within historgrams $=n$ /group. Means and SEMs above histograms $=$ mean amounts of Diets Coc and Cin eaten by observers during Steps 5 and 6 of Procedure. Means below histograms = mean amounts eaten by observers during testing (Step 9). Flags on histograms = \pm 1 SEM.

Step 9. Following the 24-h recovery period, pellets were removed from each observer's cage and each observer was offered, for $22 \mathrm{~h}$, a simultaneous choice of weighed samples of Diet Cin and Diet Coc. At the end of this $22-\mathrm{h}$ test period, the experimenter determined each observer's intake of Diet Cin and Diet Coc and calculated the percentage of Diet Cin eaten by each observer.

\section{Results and Discussion}

The main results of Experiment 1 are presented in Figure 2, which shows the mean amount of Diet Cin eaten, as a percentage of total amount ingested during testing, by observers in 0-, 1-, and 8-day delay conditions. As can be seen in Figure 2 and as statistical tests confirmed (Mann-Whitney $U$ tests; see Figure 2 for $U$ and $p$ values), regardless of the delay between (1) exposure to demonstrataors (Step 3) and (2) sampling of foods and experience of toxicosis (Steps 5, 6, and 7 in Figure 1), observers learned an aversion to that food their respective demonstrators had not eaten. Imposition of a delay, even one as great as 8 days, between extraction of information from demonstrators by observers and food sampling by observers did not have an appreciable effect on demonstrator influence on aversion learning by observers.

It is possible, although unlikely, that the results of the present experiment and the results reported in Galef (1986a) were specific to the particular diets (Diets Cin and $(\mathrm{Coc})$ used. Experiment 1 was, therefore, repeated using two different diets (Diet Ani: powdered Purina Laboratory Rodent Chow adulterated $1 \%$ by weight with ground anise seed; and Diet Mar: powdered Purina Laboratory Rodent Chow adulterated $2.4 \%$ by weight with ground marjoram). With 0 delay between Steps 3 and 5 , observers $(n=9)$ that had interacted with Diet Mar-fed demonstrators ate an average of $1.7 \%$ Diet Ani during testing (Step 9), whereas observers $(n=8)$ that had interacted with Diet Ani-fed demonstrators during Step 3 ate an average of $96.5 \%$ Diet Ani during testing (Step 9). With a 7-day delay between Steps 3 and 5, those observers $(n=9)$ that had interacted with Diet Mar-fed demonstrators during Step 3 ate $55.3 \% \pm 4.8 \%$ Diet Ani during testing (Step 9), whereas those observers $(n=9)$ that had interacted with Diet Ani-fed demonstrators during Step 3 ate $85.5 \% \pm 2.4 \%$ Diet Ani during testing (Step 9). These results both confirm and extend the generality of the main experiment. Upon experiencing toxicosis, rats are less likely to form an aversion to a previously ingested food, familiar as the result of social interaction with conspecifics that have eaten that food, than they are to form an aversion to a previously ingested food lacking socially induced familiarity. 


\section{EXPERIMENT 2}

The results both of Experiment 1 and of Galef (1986a) indicate that rats exhibit a reduced probabiltiy of forming an aversion to a food eaten by conspecifics with which they have interacted. Observer rats treat a diet eaten by others of their species as relatively "safe" (Kalat \& Rozin, 1973).

In natural circumstances, rats live in social groups whose members are likely to be feeding on a number of different foods. It seemed reasonable to ask whether an individual rat that had interacted with several conspecifics, each eating a different food, would have the capacity to store information as to the diets each of its fellows had eaten and would treat each as relatively safe. We have previously shown (Galef, 1983) that rats can distinctively encode, store, and retrieve information acquired from 3 conspecifics as to the foods they had recently eaten. In the present experiment, I asked whether the recipients of such information could use it to identify potentially safe foods.

\section{Method}

\section{Subjects}

One hundred and twelve experimentally naive 42-day-old rats acquired from Charles River, Canada (St. Constant, Quebec) served as observers, and an additional 240 rats, 63 to 84 days old, served as demonstrators. Many of the demonstrators in the present experiment had been observers in previous studies; others were born in the McMaster colony to stock acquired from Charles River, Canada. During the 2 weeks prior to the initiation of the present experiment, all demonstrators were maintained on ad-lib Purina Laboratory Rodent Chow pellets and water.

Half the demonstrators and half the observers were randomly assigned to the Diet Set 1 condition and half to the Diet Set 2 condition described below.

\section{Procedure}

Diet Set 1. The procedure of the present study was identical to that of Experiment 1 (0-delay condition) except that instead of interacting with a single demonstrator during Step 3, observers in various groups interacted with 1, 2, or 3 demonstrators. Observers interacting with a single demonstrator (1-dem group; $n=16$ ) were treated exactly like observers in the 0-delay condition of Experiment 1 , except that each observer interacted with its demonstrator for 20 rather than $30 \mathrm{~min}$. Observers interacting with 2 demonstrators during Step 3 of the experiment (2-dem group; $n=16$ ), interacted for $20 \mathrm{~min}$ with 1 demonstrator fed either Diet Cin or Diet Coc and then for 20 min with 1 demonstrator fed either Diet Mar or Diet Ani. During Step 3 of the experiment, observers interacted with 3 demonstrators (3-dem group; $n=24$ ) for 20 min each: first, with 1 demonstrator fed either Diet Cin or Diet Coc; then, with 1 demonstrator fed either Diet Ani or Diet Mar; and, last, with 1 demonstrator fed Diet NPT (Normal Protein Test Diet; Teklad, Madison, WI).

Immediately following interaction with a demonstrator or demonstrators, each observer was then subjected to Steps 5-9 of the Experiment 1 procedure, culminating with the $22-\mathrm{h}$ test of preference between Diets Cin and Coc (Step 9).

Diet Set 2. The procedure employed with observers assigned to the Diet Set 2 condition was identical to that employed with observers assigned to the Diet Set 1 condition except that: (1) during
Steps 5 and 6, respectively, all observers ate Diet Ani and then Diet Mar, (2) during Step 9, all observers were tested for their preference betwen Diets Ani and Mar, and (3) during Step 2, observers in the 1-dem group of the Diet Set 2 condition interacted with one demonstrator fed either Diet Ani or Diet Mar.

\section{Results and Discussion}

The main results of Experiment 2 are presented in Figures $3 a$ and $3 b$, which show, respectively, the outcome of the test of diet preference (Step 9) given observers assigned to Diet Sets 1 and 2. Figure 3a shows the amount of Diet Cin as a percentage of total amount eaten during testing by observers in the Diet Set 1 condition (i.e., observers that ate Diet Coc and Diet Cin during Steps 5 and 6 , respectively). As can be seen in Figure $3 a$, and as statistical tests confirmed (Mann-Whitney $U$ Tests; see Figure $3 \mathrm{a}$ for $U$ and $p$ values), those observers one of whose demonstrators had eaten Diet Coc formed an aversion to Diet Cin, and those observers one of whose demonstrators had eaten Diet Cin formed an aversion to Diet Coc.

Figure 3b shows the amount of Diet Ani as a percentage of total amount eaten during testing by observers in the Diet Set 2 condition (i.e., observers that ate Diet Ani and Diet Mar during Steps 5 and 6, respectively). As is obvious from examination of Figure $3 \mathrm{~b}$ and as statistical tests confirmed (Mann-Whitney $U$ Tests; see Figure 3b for $U$ and $p$ values), those observers one of whose demonstrators had eaten Diet Ani learned an aversion to Diet Mar; those observers one of whose demonstrators had eaten Diet Mar formed an aversion to Diet Ani. Although we did not examine the effects of prior interaction with a demonstrator fed Diet NPT on subsequent learning of an aversion to Diet NPT by subjects in the 3-dem group, it is probable that if we had done so we would have found a reduced probability of aversion learning to Diet NPT in observers exposed to Diet NPT-fed demonstrators. Observers can extract information from demonstrators fed Diet NPT that influences their subsequent learning of aversions to Diet NPT (Galef, 1985, 1986b, 1987). During Step 3, observers in the 3-dem groups did not know which diets they would be exposed to in Steps 5 and 6 or which diets they would have to choose between in Step 9. Thus, from the subjects' perspective, the experimenter was randomly sampling among the diets fed to demonstrators in testing the effects of demonstrators' diets on aversion learning by observers. The fact that observers showed reduced aversion learning to those diets selected for testing strongly suggests that observers would have exhibited a similar response to that diet not selected for testing. Thus, although, strictly speaking, it has been demonstrated only that observer rats can use information from 2 demonstrators fed different diets in identification of toxic foods, there is every reason to believe that they can use information from 3 demonstrators as well.

The results of the present experiment show that rats interacting with 3 demonstrators, each fed a different food, 

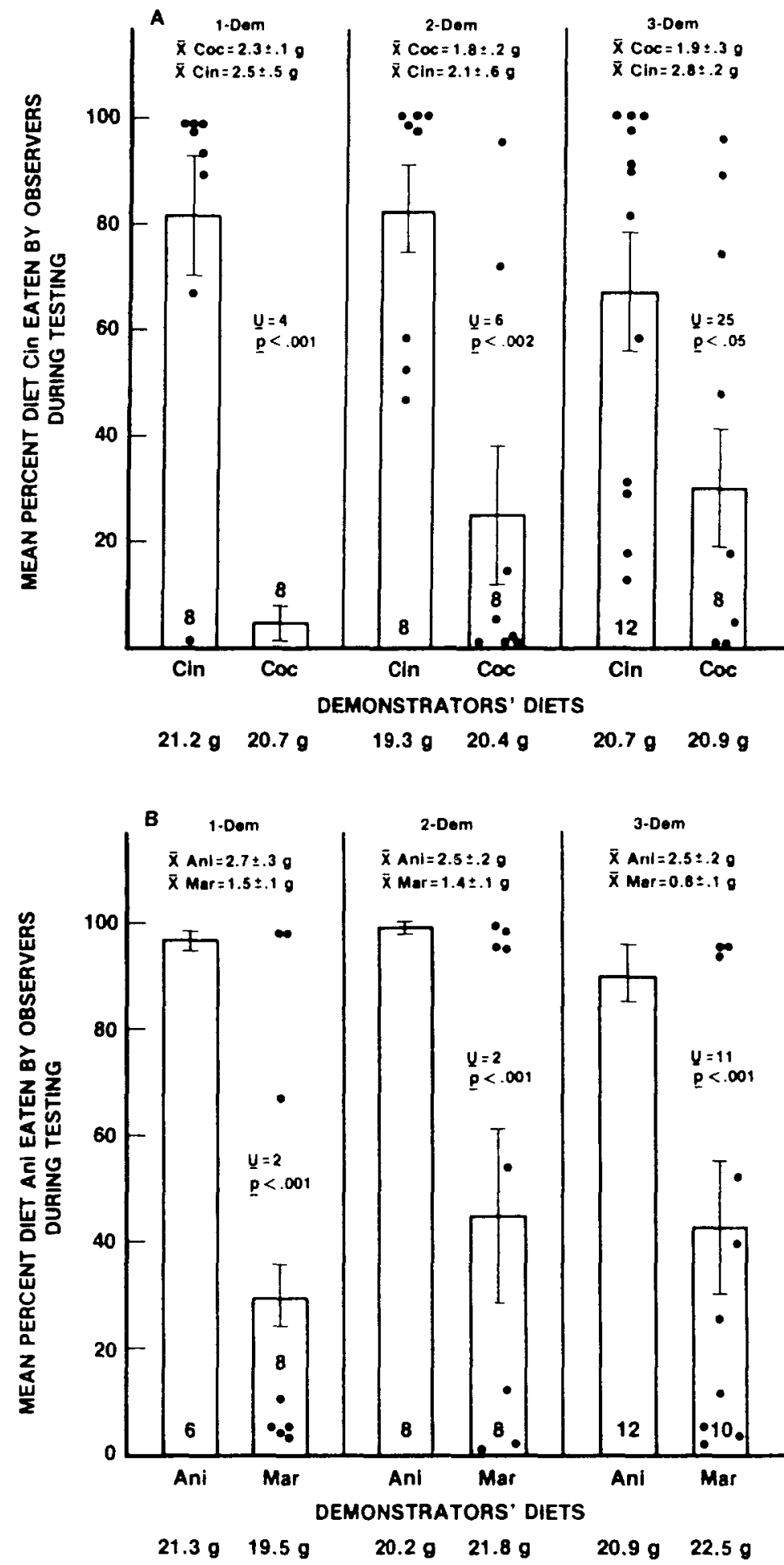

Figure 3. Mean amount of Diet Cin (3A) or Diet Ani (3B) eaten by observers, as a percentage of total amount consumed during testing (Step 9 of Procedure). Numerals within histograms $=n$ /group. Means and SEMs above histograms = mean amounts of Diets Coc and Cin (3A) or Diets Ani and Mar (3B) eaten by observers during Steps 5 and 6 of Procedure. Means below histograms = mean amounts eaten by observers during testing (Step 9). Points = individual data points. Flags on histograms $= \pm 1 S E M$. 
subsequently exhibit a reluctance to form an aversion to each of the diets eaten by those demonstrators. Rats living in social groups in nature have the capacity to store information as to the foods eaten by at least 3 of their fellows and will treat those foods as relatively safe should they eat more than one novel food and become ill.

\section{GENERAL DISCUSSION}

In a series of studies conducted over the past several years, evidence has been provided that naive rats are able to extract information from recently fed conspecifics as to the diets those conspecifics have eaten. Such socially acquired information as to the foods that others are eating can play a significant role in the formation and maintenance of learned aversions: (1) A substantial proportion of rats that have acquired an aversion to a diet, as the result of association of that diet with toxicosis, will abandon that aversion following exposure to conspecifics that have eaten the averted diet (Galef, 1985, 1986b). (2) Both the present results and those of Galef (1986a) indicate that interaction with a conspecific that has eaten some diet interferes with the subsequent formation of an aversion to that diet.

A rat living as a member of a social group, as rats live in extralaboratory environments, should be less likely to form an aversion to foods eaten by conspecifics than to other novel foods it ingests. Both an individual's experience of the postingestional consequences of ingesting dietary items and the information it acquires as to the foods others in its social group are eating are important determinants of the aversions that rats learn.

\section{REFERENCES}

GALEF, B. G., JR. (1983). Utilization by Norway rats ( $R$. norvegicus) of multiple messages concerning distant foods. Journal of Comparative Psychology, 97, 364-371.

GaLeF, B. G., JR. (1985). Socially induced diet preference can partially reverse a $\mathrm{LiCl}$-induced diet aversion. Animal Learning \& Behavior, 13, 415-418.

GALEF, B. G., JR. (1986a). Social identification of toxic diets by Norway rats. Journal of Comparative Psychology, 100, 331-334.

GALEF, B. G., JR. (1986b). Social interaction modifies learned aversions, sodium appetite, and both palatability and handling-time induced dietary preference in rats (Rattus norvegicus). Journal of Comparative Psychology, 100, 432-439.

GALEF, B. G., JR. (1987). Social blockade of taste-aversion learning in Norway rats (Rattus norvegicus): Preventing development of "food phobias." Manuscript submitted for publication.

GAlef, B. G., JR., \& STEIN, M. (1985). Demonstrator influence on observer diet preference: Analysis of critical social interactions and olfactory signals. Animal Learning \& Behavior, 13, 31-38.

GAlEF, B. G., JR., \& WigmoRE, S. W. (1983). Transfer of information concerning distant foods in rats: A laboratory investigation of the 'information centre' hypothesis. Animal Behaviour, 31, 748-758.

KALAT, J. W., \& RozIN, P. (1973). "Learned safety" as a mechanism in long-delay taste-aversion learning in the rat. Journal of Comparative \& Physiological Psychology, 83, 198-207.

Posadas-AnDrews, A., \& RoPER, T. J. (1983). Social transmission of food preferences in adult rats. Animal Behaviour, 31, 265-271

RevUsKY, S. H., \& BEDARF, E. W. (1967). Association of illness with prior ingestion of novel foods. Science, 155, 219-220.

(Manuscript received September 29, 1986; revision accepted for publication January 16, 1987.) 